Int. J. Speleol. 18 3-4 (1989), pp. 117-144, 1 f.t.

\title{
I CAMPI SOLCATI DI BORGO GROTTA GIGANTE NEL CARSO TRIESTINO ${ }^{\circ}$
}

\author{
Franco Cucchi ${ }^{\star}$, Nadia Radovich \& Ugo Sauro ${ }^{*}$
}

\section{PREMESSA}

Nei pressi di Borgo Grotta Gigante, nell'area localmente nota come "Gadna Griza" (griza delle vipere), si trovano alcuni dei campi solcati più belli del Carso Triestino. L'area più interessante si trova nel quadrato chilometrico UTM 33T VL 0362, tavoletta Poggioreale del Carso (40A II SO).

Per raggiungere gli affioramenti più suggestivi si percorre la strada comunale che lascia Borgo Grotta Gigante verso Ovest in direzione Prosecco. Un centinaio di metri dopo il sottopassaggio della ferrovia si imbocca sulla destra una carrareccia e la si segue per circa $300 \mathrm{~m}$ in direzione Nord, svoltando poi a sinistra (verso Ovest) lungo un sentiero fiancheggiato a sinistra da alberi ed arbusti e a destra da un'ampia radura. Si arriva così al bordo settentrionale di una grande dolina chiamata localmente "Skoludnjek": si tratta di una dolina appena asimmetrica, con il fianco nordorientale un poco più ripido, profonda una quarantina di metri e del diametro di circa 250 metri.

Il bordo orientale della dolina è particolarmente ricco di affioramenti rocciosi, consistenti in testate di strato allungate alcune decine di metri, con larghezze comprese fra un paio e la decina di metri. Sulle superfici di strato, debolmente inclinate verso Sud, abbondano le forme carsiche minori e danno origine ai campi solcati fra i più belli ed estesi del Carso Triestino, micropaesaggi in roccia particolarmente suggestivi.

* Istituto di Geografia e Oceanografia dell'Università di Messina

* * Dipartimento di Geografia dell'Università di Padova

- Ricerca eseguita con contributo del Ministero Pubblica Istruzione 40\% “Morfodinamica e cronologia del carsismo delle Alpi Meridionali"'.

Il lavoro è frutto di collaborazione fra i tre Autori: $F$. Cucchi ha curato in particolare l'inquadramento geologico, N. Radovich il rilevamento dei campi solcati e la raccolta dei dati morfometrici relativi. Tutti e tre gli Autori hanno partecipato alla discussione relativa all'analisi morfologica e all'elaborazione dei dati. 
I rilievi in roccia sono nettamente asimmetrici, presentando su un lato un esteso versante piano e poco ripido, costituito da una superficie di strato, e sull'altro una ripida scarpata che taglia gli strati. È evidente come lo sviluppo di questi rilievi sia stato condizionato dalla struttura geologica ed in particolare dalla presenza di calcari compatti che hanno determinato l'individuazione di un rilievo di tipo monoclinale, dato da una serie di caratteristiche "microcueste". Poiché l'immersione prevalente degli strati è verso Sud, con inclinazioni comprese fra $4^{\circ}$ e $14^{\circ}$, i versanti di faccia di strato sono esposti verso mezzogiorno mentre quelli di testata guardano verso settentrione.

\section{ASPETTI DELLA RICERCA SUI CAMPI SOLCATI}

I campi solcati costituiscono dei complessi microambienti il cui studio ha fornito informazioni di particolare interesse per la comprensione della storia recente degli ambienti, delle interrelazioni fra processi atmosferici, processi morfogenetici e dinamici in ambiente carsico e sviluppo di comunità di microorganismi pionieri su roccia nuda. Se le ricerche sui Karren sono ormai numerose, certamente resta ancora molto da fare proprio nel campo della ricerca interdisciplinare di tipo ambientale e microambientale.

I campi solcati di Borgo Grotta Gigante sono già stati oggetto di ricerche scientifiche. In particolare hanno fornito il materiale per la prima ricerca di analisi morfometrica su alcuni tipi di Karen (BELLONI \& OROMBELLI, 1970), sono stati oggetto di numerose ricerche sui rapporti fra litologia e forme (FORTI F., 1971), e sono attualmente stazione per la misura diretta della velocità della denudazione carsica (erosione chimica) (FORTI, 1983; CUCCHI \& FORTI, 1987). Da queste ultime si evince che nell'area la superficie dei calcari attualmente è soggetta ad "erosione" per uno spessore di circa $0,03 \mathrm{~mm} /$ anno.

Scopo della presente nota non è quello di riassumere i lavori citati, cui si rimanda, ma piuttosto di considerare alcuni di questi micropaesaggi nel loro complesso, per cogliere le interrelazioni fra le varie forme e per tentarne un'interpretazione evolutiva, ed anche per fornire ulteriori informazioni di tipo morfologico sulle vaschette di corrosione, considerate come forme chiave per la comprensione dei paesaggi.

Per questo si sono raccolti dati sul terreno e si è proceduto alla costruzione di schizzi geomorfologici a grandissima scala di alcuni campi solcati (RADOVICH, 1988).

Questi schizzi sono stati ricavati da una copertura stereoscopica di foto zenitali ottenute con il metodo illustrato in SAURO $(1975,1977)$, cioè 
scattando strisciate di foto con macchina fotografica munita di obbiettivo grandangolare, posta su di un supporto metallico dell'altezza di cinque metri. Gli schizzi geomorfologici ottenuti forniscono un'ottima base per la lettura di questi micropaesaggi in roccia.

Sono stati presi in considerazione quattro affioramenti denominati A, B, C, C': di questi A e B sono allineati lungo la medesima bancata nell'area compresa fra la strada che collega Borgo Grotta Gigante a Prosecco ed il bordo SE della grande dolina sopracitata. I campi solcati $\mathrm{C}$ e C' sono invece situati più a Nord, poco a NE della medesima dolina e costituiscono un unico esteso affioramento.

\section{LE PICCOLE FORME DI CORROSIONE PIÜ RAPPRESENTATE}

Già da una prima analisi di questi campi solcati è possibile osservare come vi siano ben rappresentate le piccole forme di corrosione più comuni ed in particolare:

I) le scannellature carsiche (Rillenkarren): piccoli solchi rettilinei a sezione semicircolare, profondi da pochi millimetri ad oltre un centimetro, larghi fra $0,5 \mathrm{~mm}$ e $3-4 \mathrm{~cm}$ e lunghi fra pochi centimetri e circa un metro. Queste scannellature non sono mai isolate ma raggruppate in complessi di forme elementari contigue e subparallele, separate fra di loro da crestine aguzze, che talora si biforcano verso valle delimitando la testata di un'altra scannellatura. I complessi possono presentare forme a fascio, a pettine, a penna e ad isola. La massima evidenza di queste forme si ha su superfici con inclinazioni comprese fra $10^{\circ}$ e $60^{\circ}$, tuttavia è possibile trovarle anche su superfici suborizzontali e subverticali.

È opinione comune che queste forme siano le prime ad individuarsi a valle di microspartiacque in roccia, ove durante lc piogge le gocce d'acqua possono impattare direttamente con la roccia frammentandosi in minute goccioline che finiscono con l'alimentare dei filetti di corrente.

Ove, a maggior distanza dallo spartiacque, un film d'acqua di maggior continuità e spessore copre interamente la roccia i filetti di corrente perdono la loro individualità e queste forme si attenuano sino ad esaurirsi e a venire sostituite da una superficie più liscia (Ausgleichflache di BOEGLI, 1960) ove il processo della corrosione opera in modo pressoché omogeneo;

II) i solchi a doccia (Rinnenkarren): solchi decisamente più grandi dei precedenti, larghi più di $4-5$ centimetri, profondi più di $2-3 \mathrm{~cm}$ e lunghi più di un metro (le larghezze e profondità possono arrivare anche a qualche decimetro e le lunghezze a qualche decina di metri). Si tratta di "rivo- 


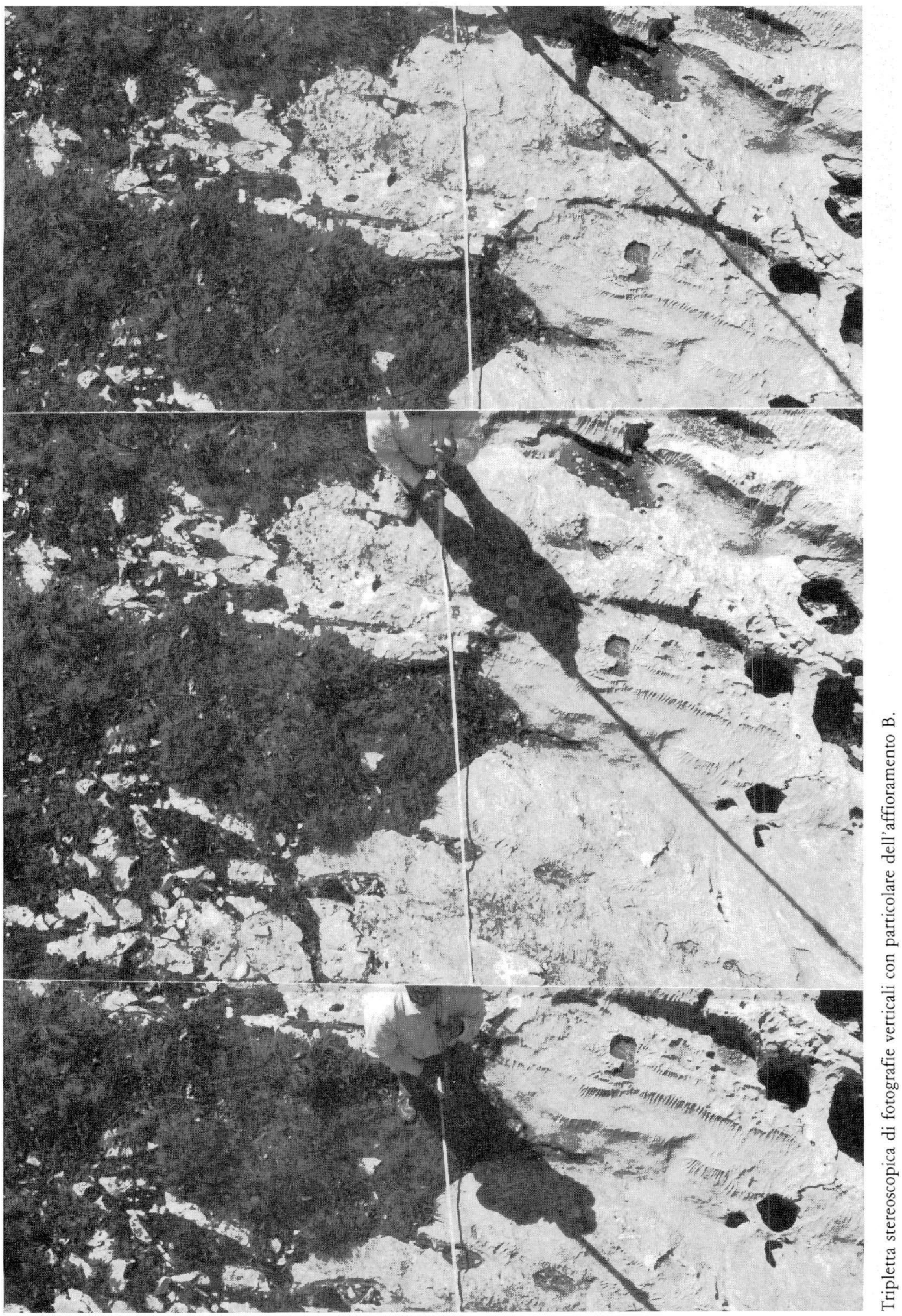



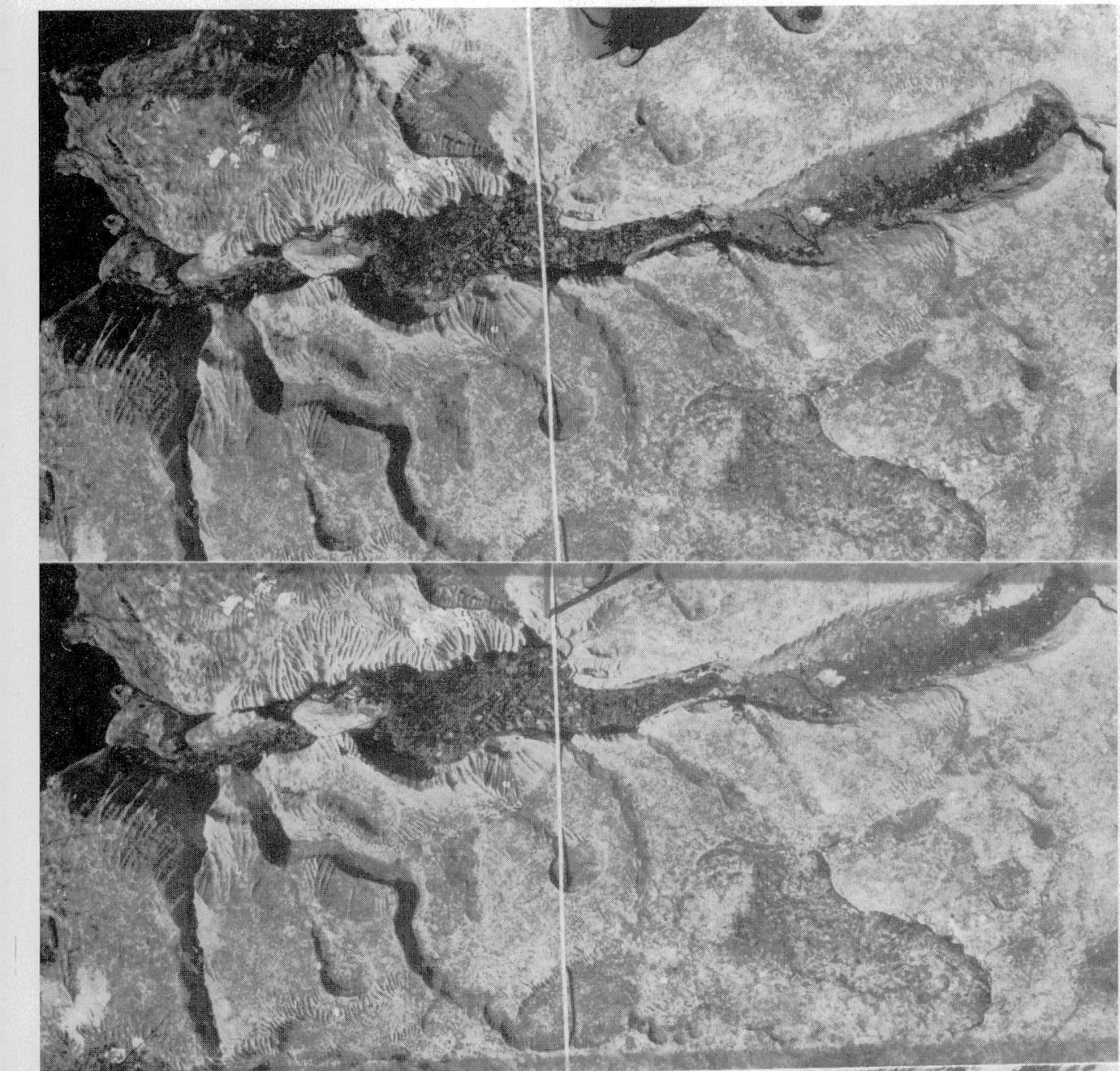

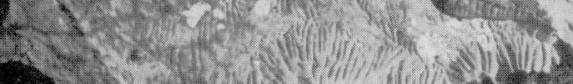

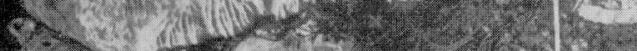

$22 x-5$

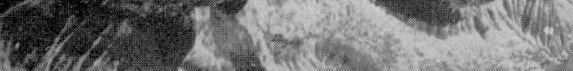

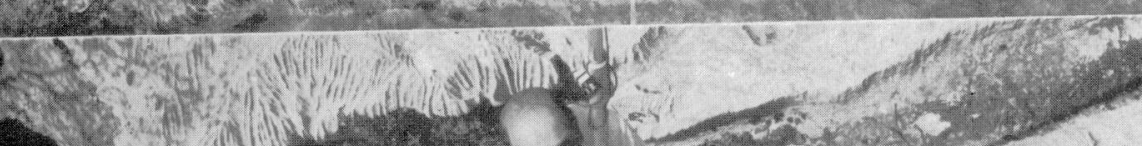

+2.

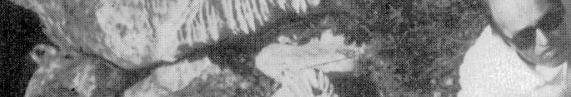

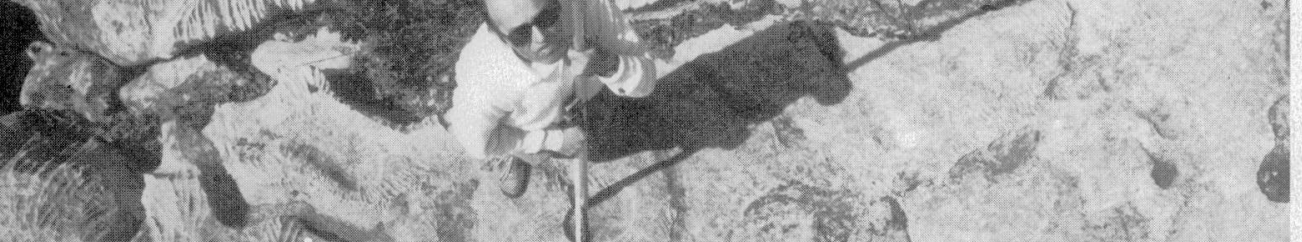

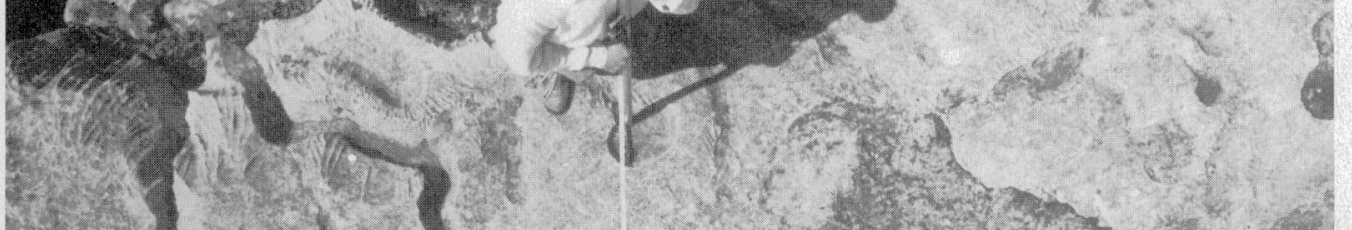

(4.






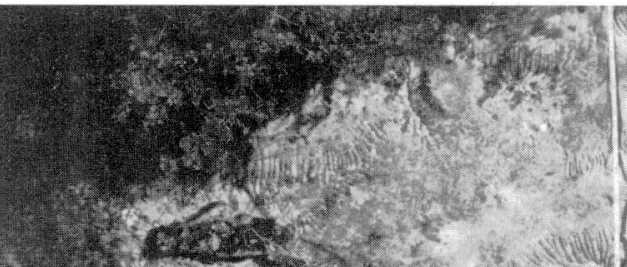


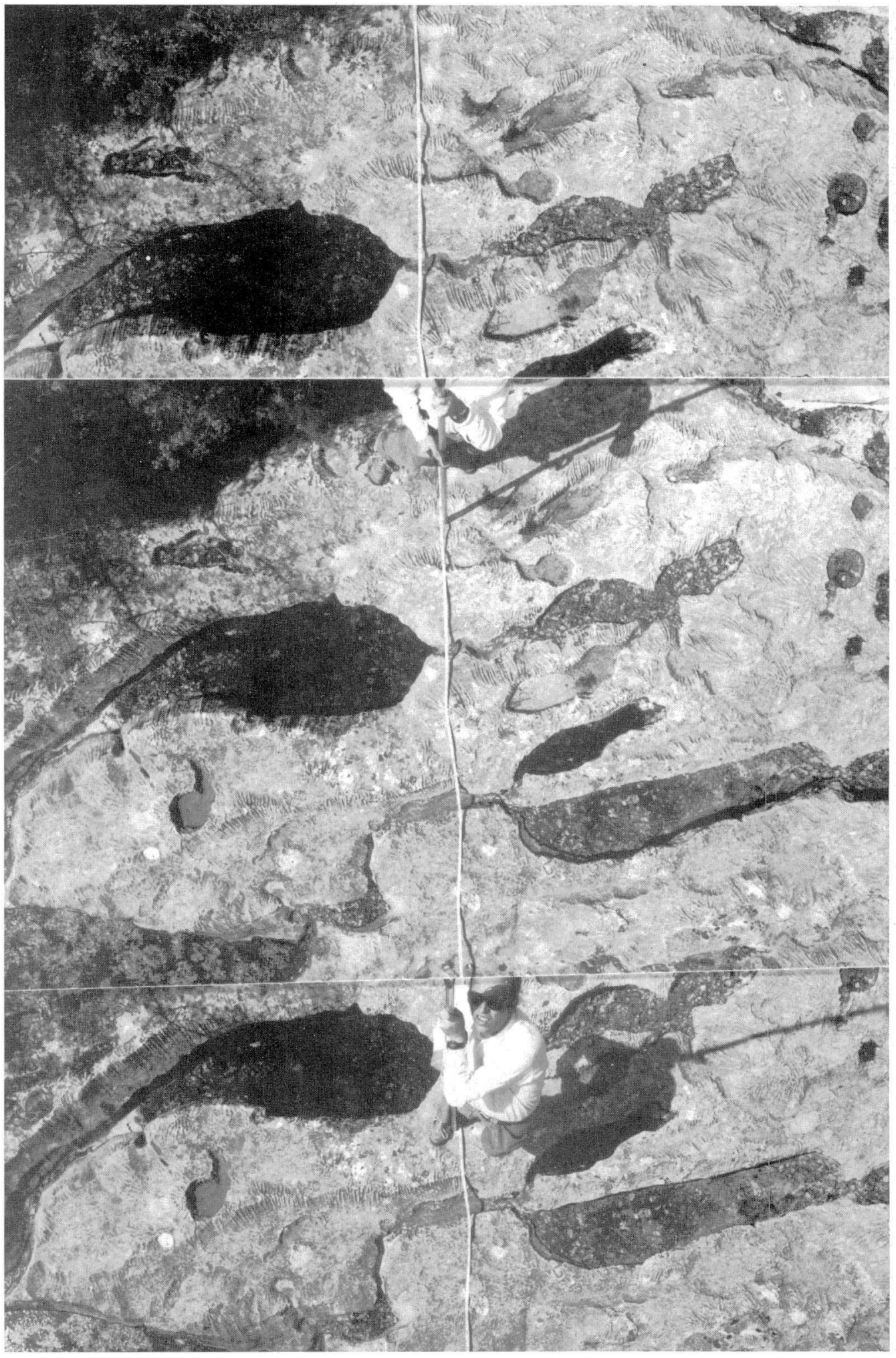

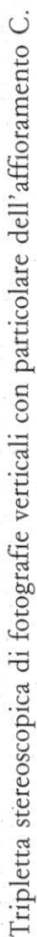




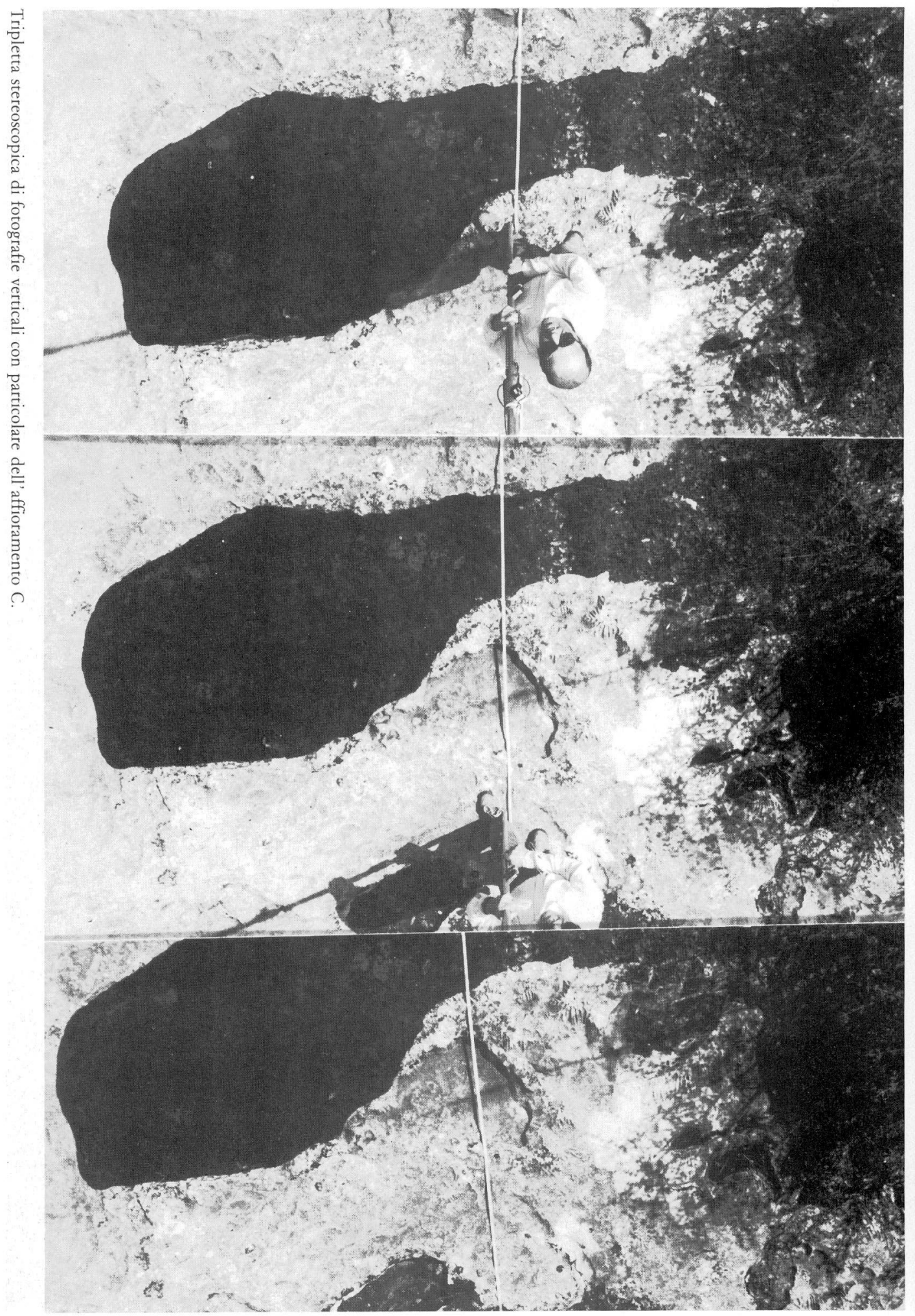


li" carsici di tipo "hortoniano" (FORD \& WILLIAMS, 1989), cioè comparabili ai rivoli dell' erosione normale su rocce incoerenti non carsiche. Questi solchi, che seguono le linee di massima pendenza, solo di rado formano fasci strettamente continui e sono separati da creste aguzze. Essi si presentano con una grande varietà di aspetti, distinguibili soprattutto per i caratteri delle sezioni trasversali, il tipo di sviluppo planimetrico e la morfologia del fondo del solco. Nell'area in studio sono presenti solchi con sezioni sia a doccia semplice (sezione trasversale ad U), sia a doccia sottoescavata, a doccia svasata e del tipo a piccola gola. Si tratta di solchi che si originano o da zolle di suolo o da vaschette carsiche. Nel primo caso si tratta di solchi a doccia da "humus" (Humusrinnenkarren) che in genere posseggono una sezione a doccia sottoescavata almeno nei settori in cui hanno potuto formarsi zolle di suolo pioniero; nel secondo si tratta di emissari di troppo pieno dell'acqua di conche chiuse, cioè di minuscoli laghetti temporanei che possono traboccare durante le piogge alimentando piccole correnti di acque ricche di microorganismi pionieri.

Dal punto di vista genetico si ritiene che i solchi a doccia si formino dove durante le piogge si individuano piccole correnti d'acqua del tipo "rivolo", cioè dove nell'ambito di uno strato laminare, l'acqua converge aumentando di spessore e formando una corrente turbolenta più veloce. Tuttavia nel caso dei solchi da humus e degli emissari delle vaschette, la corrente viene alimentata da un "serbatoio" situato a monte che può essere una vaschetta o una zolla di suolo; in questo senso FORD \& LUNDBERG (1987) definiscono queste forme "solchi di decantazione" (decantation runnels). Qui inoltre l'azione morfogenetica del rivolo è determinata, in parte, anche dalle attività biologiche delle comunità pioniere dell'acqua della vaschetta o di quella contenuta nel suolo e si tratta quindi anche di solchi biocarsici.

III) le vaschette di corrosione, indicate con il termine internazionale di "Kamenitze": si tratta di depressioni chiuse in roccia, generalmente con forma subcircolare o ellittica e con fondo subpianeggiante. Nelle forme medie e grandi le pareti sono generalmente strapiombanti. Sono stati descritti diversi tipi di vaschette fra cui ricordiamo:

a) le coppelle o ciottolette, forme embrionali di modesto diametro, poco profonde e svasate verso l'alto,

b) le kamenitze a vasca semplice, caratterizzate da forma generalmente ellittica, fondo piatto, fianchi sottoescavati e solco emissario,

c) le kamenitze a coppa, varietà del tipo precedente di forma circolare, molto profonde, con emissario poco profondo, 


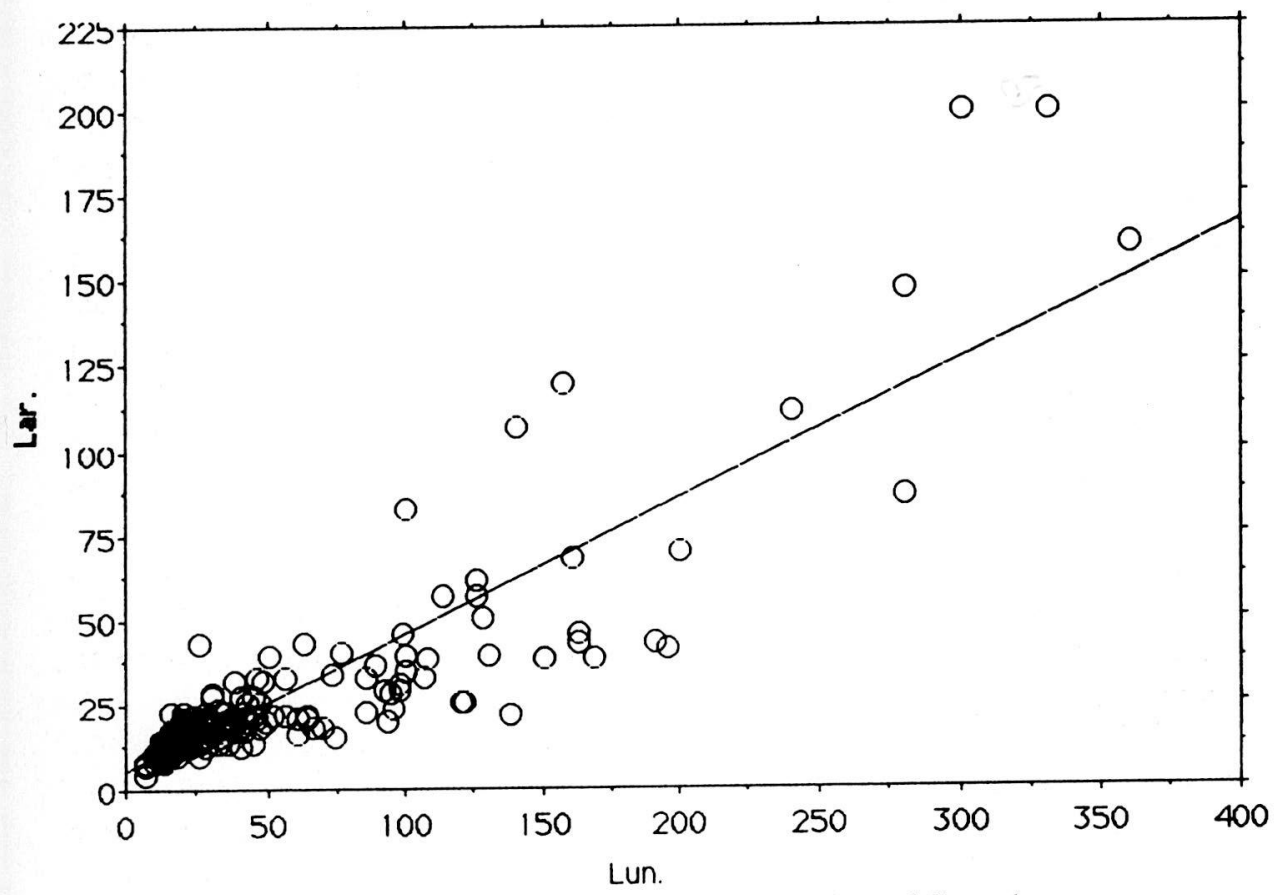

Fig. 1 - Grafico che illustra la relazione fra la lunghezza e la larghezza delle vaschette.

d) le kamenitze policicliche, altra varietà delle kamenitze a vasca, in cui sui fianchi si riconoscono più livelli di cornici che si sono individuati in relazione a variazioni del livello di soglia,

e) le kamenitze a nido, depressioni che ospitano sul fondo frammenti di roccia, contenuti in cavità della forma degli stessi frammenti, e che richiamano quindi per analogia nidi contenenti uova,

f) le kamenitze lobate, che possono derivare dalla coalescenza di più forme adiacenti o dall'allargamento di parti del perimetro in direzioni preferenziali,

g) le kamenitze innicchiate sul fondo di grandi solchi,

h) le kamenitze con depositi sciolti fini e qualche ciottolo sul fondo interessato di conseguenza da sottoescavazione puntuale sottocutanea.

In ambienti particolari quali quelli costiero e fluviale si possono osservare anche delle forme di transizione fra delle kamenitze e delle marmitte di evorsione, cioè delle kamenitze-marmitte.

Le kamenitze sono state descritte come delle forme biocarsiche o fitocarsiche (PERNA, 1974; PERNA \& SAURO 1978), o come forme essen- 
zialmente dissolutive (FORTI, 1971). Per i primi Autori si tratterebbe di forme che si individuano in seguito all'attacco della roccia da parte di comunità di microorganismi pionieri che ne facilitano la dissoluzione permettendo la formazione di una conca chiusa ove si può raccogliere acqua che a sua volta crea un ambiente favorevole a comunità biologiche via via meglio differenziate che accelerano la corrosione. Che la presenza d'acqua acceleri la corrosione è provato, tra l'altro, dal fatto che i fianchi siano generalmente strapiombati ed il fondo suborizzontale: infatti la corrosione si dimostra più attiva dove l'acqua ristagna più a lungo, cioè in prossimità del fondo.

Secondo FORTI è semplicemente e solamente l'acqua, statica su superfici poco o nulla inclinate, ad originare la conca chiusa iniziale che a sua volta favorisce ulteriori incrementi di acqua statica. La dissoluzione approfondisce dapprima, allarga poi la conca con forme direttamente legate alle caratteristiche litologiche, petrografiche e strutturali della roccia. L'attività dissolutiva dell'acqua (incrementata eventualmente da fatti biologici e/o

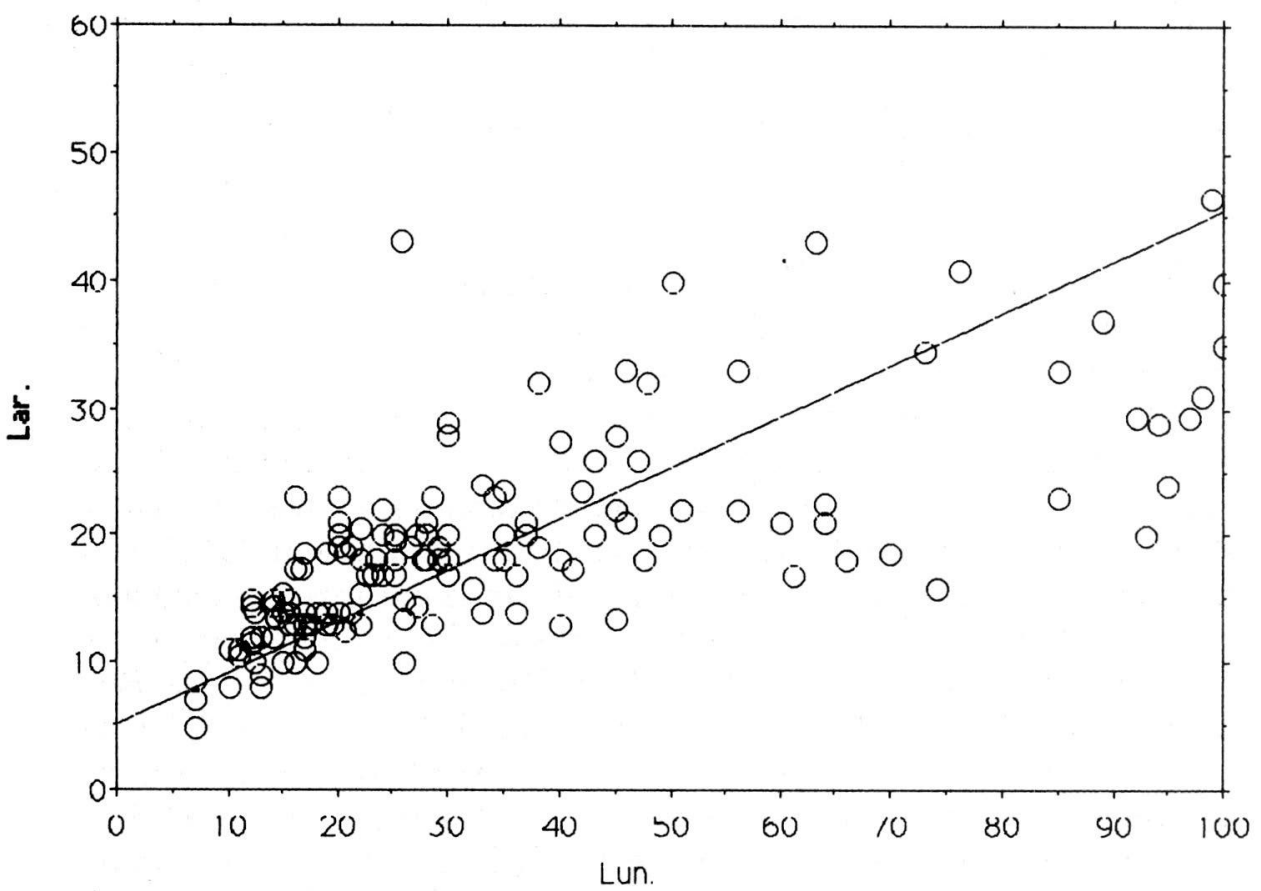

Fig. 2 - Ingrandimento di parte del grafico precedente da cui si coglie l'addensamento della popolazione intorno a valori compresi fra 15 e $40 \mathrm{~cm}$ per la lunghezza e 10 e $20 \mathrm{~cm}$ per la larghezza. 


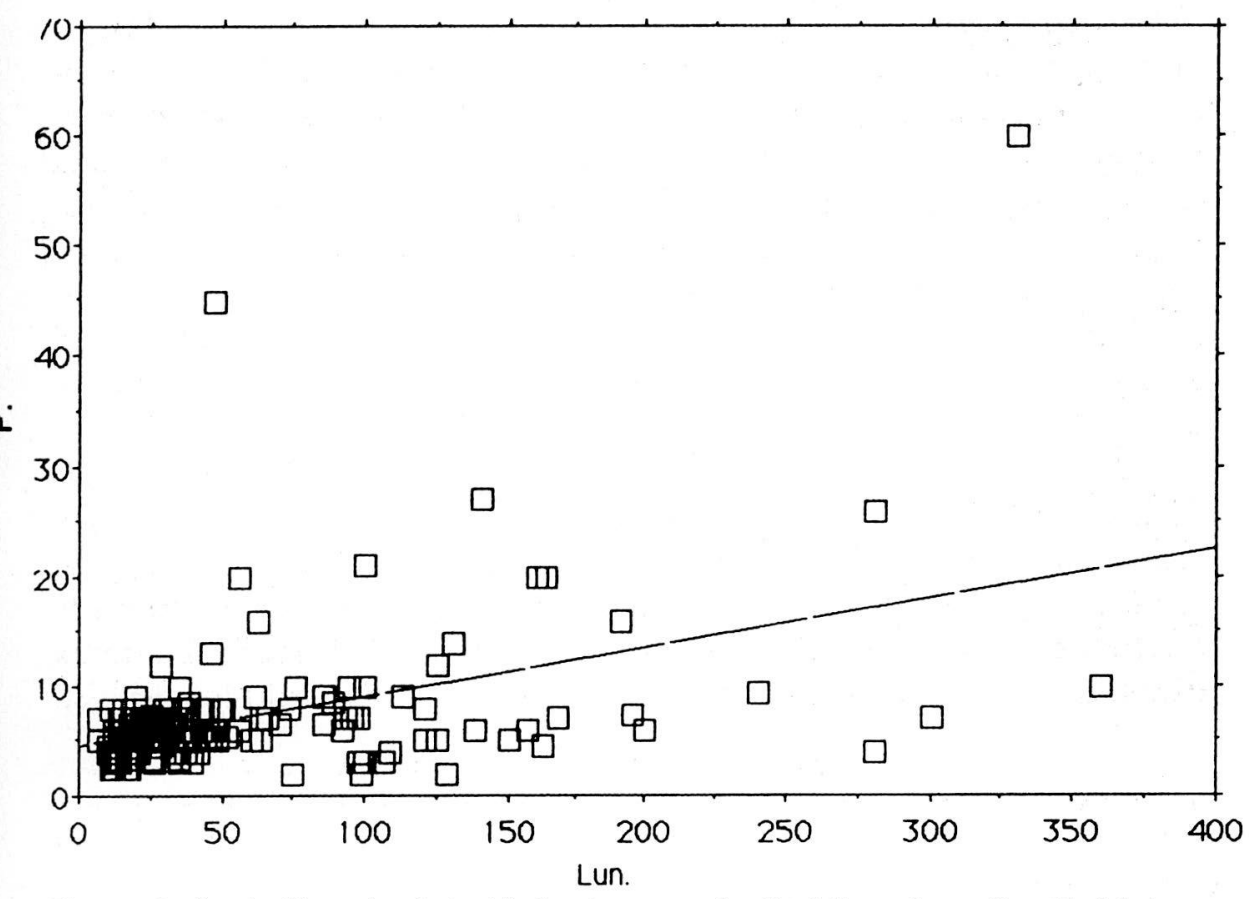

Fig. 3 - Grafico che illustra le relazioni fra lunghezza e profondità delle vaschette. Si noti la debole relazione che lega le dimensioni della vaschetta con la profondità.

vegetali) è massima sui fianchi della vaschetta ove si hanno scambi ionici all'interfaccia aria-acqua, minima sul fondo ove si raggiunge ben presto una condizione di sovrasaturazione e staticità.

IV) I fori ed i crepacci carsici, forme che intaccano profondamente la compagine rocciosa in forma di cavità tubulari più o meno regolari e di fessure, o crepacci o corridoi che spesso si restringono verso il basso o si chiudono in corrispondenza di superfici di interstrato. Si tratta di Karren non più strettamente superficiali ma di forme di transizione verso le cavità ipogee. I caratteri morfologici di alcuni campi solcati dimostrano che queste forme non sono solo di tipo "diretto", sviluppatesi cioè dalla superficie esterna verso l'interno della massa rocciosa, ma che una volta individuate le prime forme esse tendono a propagarsi con meccanismi di tipo laterale ed inverso: come una carie esse si diffondono per meccanismi di corrosione accelerata innescati dalle azioni biocarsiche facilitate dalla condensazione di umidità sulle pareti e dalla penetrazione al loro interno di sostanze organiche (SAURO, 1974). Il rapido propagarsi di queste forme all'interno 
di un campo solcato può provocare una distruzione di questo con sviluppo di una copertura di suolo vegetale.

Nell'ultima classificazione delle forme di Karren (FORD \& WILLIAMS, 1989), vengono distinte le "forme circolari-piane" fra cui vengono considerate le Kamenitze, le "forme lineari controllate dalla fratturazione" fra cui fori e crepacci, le "forme lineari controllate dall'idrodinamica" fra cui le scannellature ed i solchi, e le "forme poligenetiche".

\section{LE CARATTERISTICHE GEOLOGICHE DELL'AREA}

La dolina e il suo bordo sono caratterizzati dall'affioramento di rocce calcaree appartenenti al "Membro di Borgo Grotta Gigante" della, per ora informale, "Formazione dei Calcari del Carso Triestino" (CUCCHI, PIRINI RADRIZZANI \& PUGLIESE, 1987). Si tratta di calcari puri, compatti, micritici, talora ricchi di frammenti di resti organici, sedimentatisi in un articolato ambiente di scogliera tardocretacica.

Dal punto di vista litologico si ha così nella zona una prevalenza di micriti fossilifere; si tratta di mudstone, spesso bioturbato, con rarissime Miliolidae alternate a biomicriti; o di wackestone con Miliolidae, Lituoli-

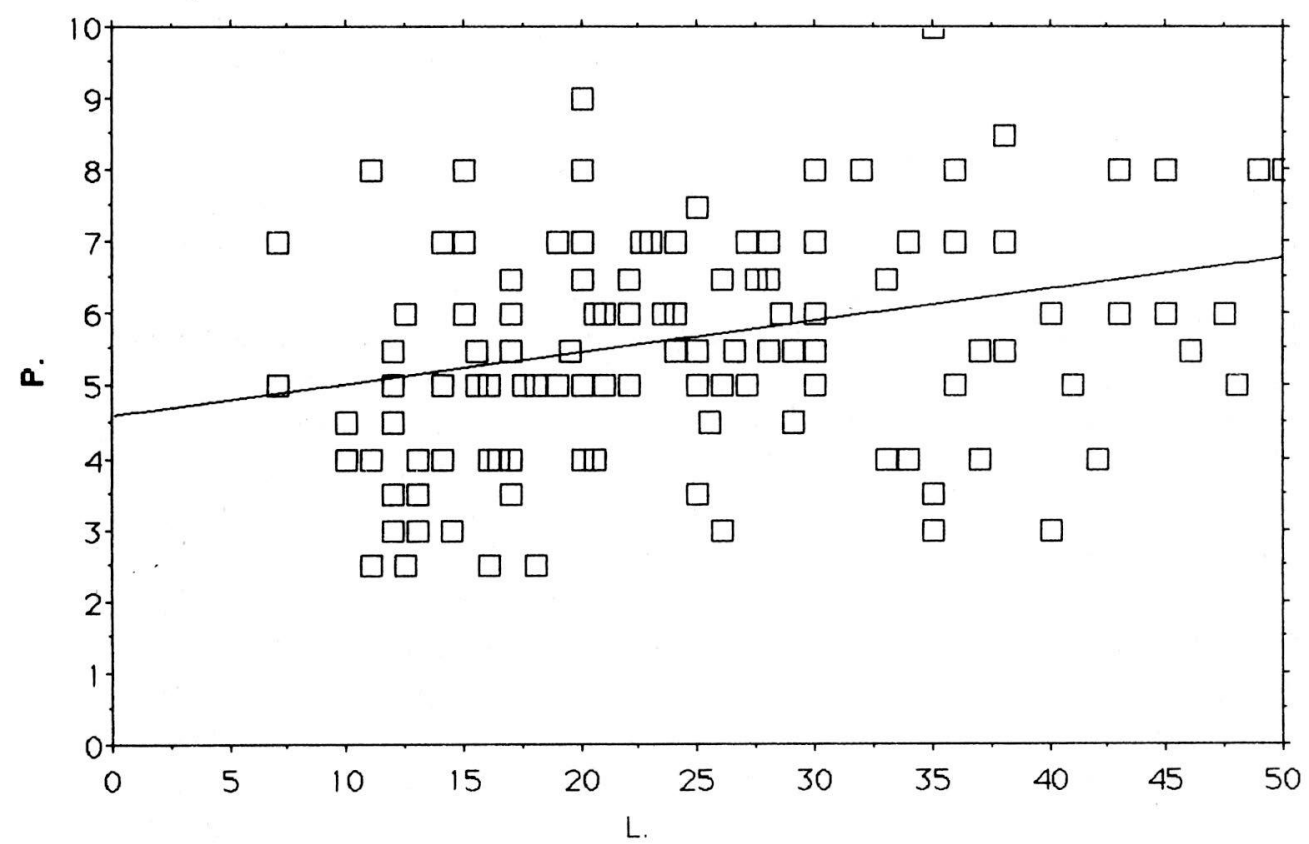

Fig. 4 - Ingrandimento di una parte del grafico precendente. 


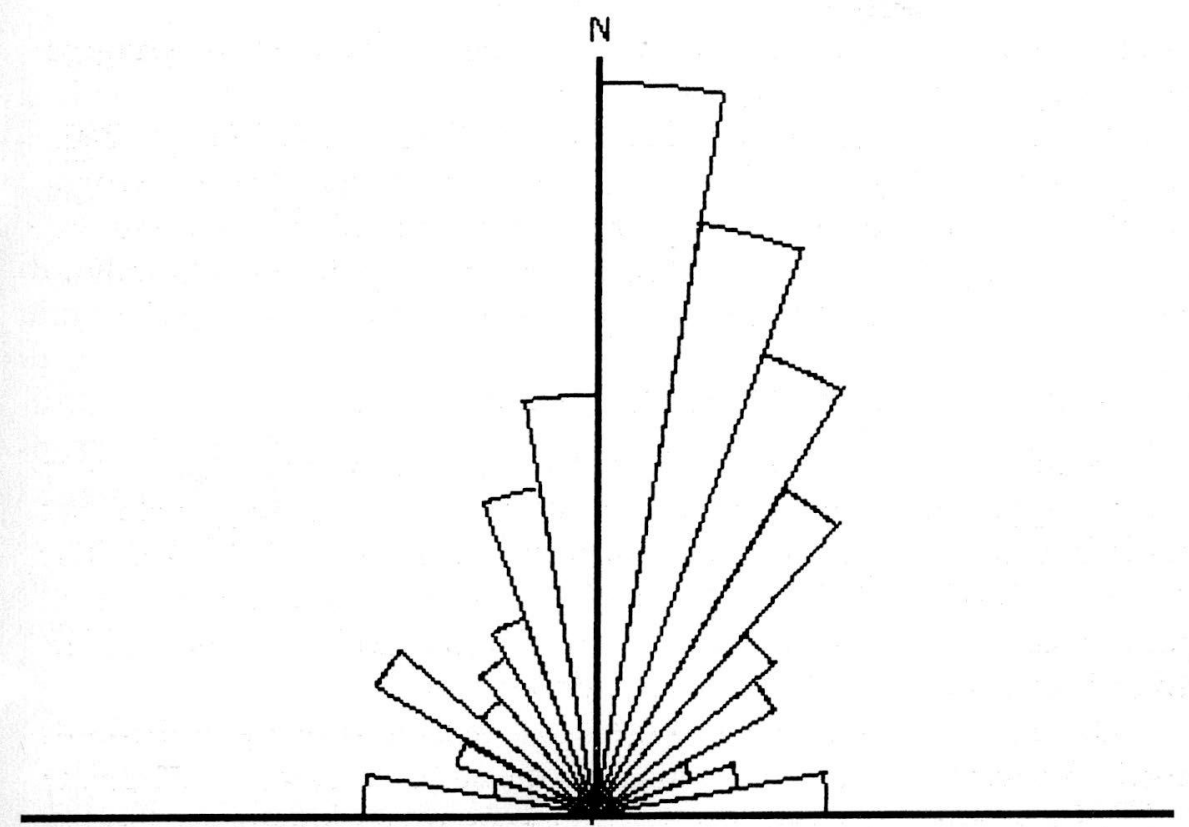

Fig. 5 - Direzioni degli assi maggiori delle vaschette verso i primi tratti dei canali di scarico. Si noti la prevalenza di direzioni comprese fra $180^{\circ}-240^{\circ}$. Il diagramma stellare è del tipo ad aree proporzionali (la scala lungo i raggi equivale alla radice quadrata della percentuale).

dae, Ophtalmiidae, Aeolisaccus Kotori, Textulariidae, Thaumatoporella, ed altri Foraminiferi; o di packstone con bioclasti (Rudiste), rare Lituolidae e Thaumatoporella Parvovesiculifera.

Il ritmo della stratificazione è variabile da alcuni centimetri al metro; i piani di strato sono solitamente netti ma non mancano episodi di stratificazione indistinta che portano le bancate ad avere talora potenza di 100$150 \mathrm{~cm}$. La fratturazione, comunque non intensa, ha frequenza legata allo spessore degli strati: là dove questi sono potenti alcune decine di centimetri la frequenza è da decimetrica a metrica, là dove la stratificazione è centimetrico-decimetrica, decimetrico è il ritmo di fratturazione.

Dal punto di vista strutturale, la stratificazione ha direzione EstOvest (con lievi variazioni) e inclinazione da $1^{\circ}$ a $14^{\circ}$ verso Sud. Il sistema di fratturazione è semplice, con famiglie subverticali orientate preferenzialmente N-S, NE-SW, E-W, NW-SE; fra esse la prima può definirsi la prevalente (CUCCHI et alii, 1983).

L'area fa parte di una zona ad intensissima carsificazione (la "carsificabilità', nel senso di FORTI - 1979, è massima, cioè di grado 5), tuttavia 
le cavità rilevate negli immediati dintorni dei campi solcati, non sono numerose: 8 in circa mezzo chilometro quadrato, essenzialmente brevi pozzi impostati su direttrici N-S.

\section{ALCUNI ASPETTI MORFOLOGICI DEI CAMPI SOLCATI}

Come abbiamo visto, i campi solcati studiati occupano i versanti di stratificazione di microcueste. Vi prevalgono le forme sopra descritte ed in particolare le kamenitze, i solchi a doccia e le scannellature. Dal punto di vista percentuale l'area occupata da queste forme nell'affioramento C, (superficie totale di $113,8 \mathrm{~m}^{2}$ ) è rispettivamente del $11 \%, 7 \%$, e $16 \%$, mentre nell'affioramento C' (area totale di $86,27 \mathrm{~m}^{2}$ ) è rispettivamente del $7 \%, 13 \%$ e $17 \%$.

Sono state oggetto di analisi morfometrica le 184 kamenitze presenti negli affioramenti cartografati o in prossimità di questi, delle quali sono forniti dati dimensionali e caratteristiche nelle tabelle.

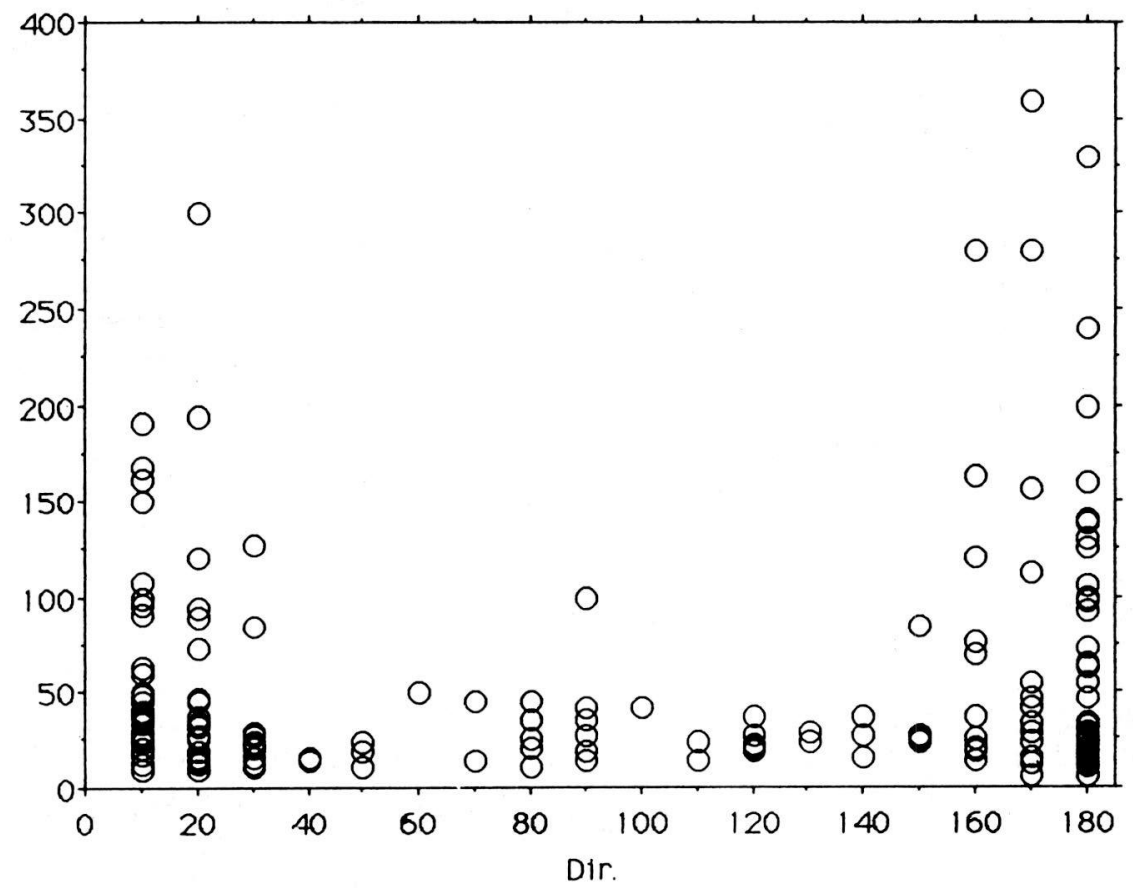

Fig. 6 - Grafico che mostra le interrelazioni fra la direzione degli assi maggiori e le lunghezze delle kamenitze; si nota che il campo di distribuzione mostra una forma a campana (le due metà della campana sono scomposte in modo speculare sui due lati del grafico), la quale evidenzia come le vasche più lunghe presentino orientamenti entro il settore fra $\mathrm{N} 20^{\circ} \mathrm{W}\left(340^{\circ}\right)$ e $\mathrm{N} 20^{\circ} \mathrm{E}$ $\left(20^{\circ}\right)$. 


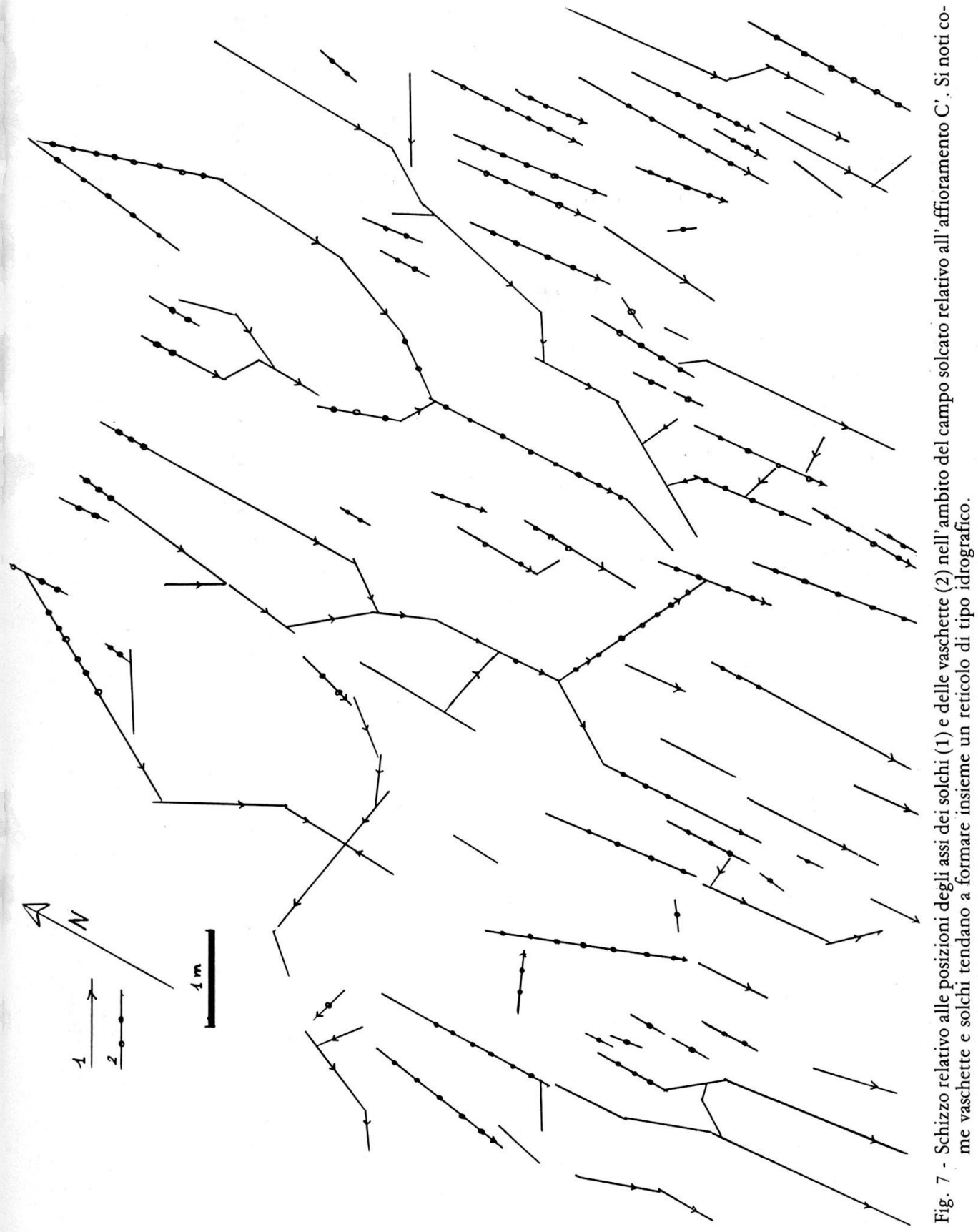




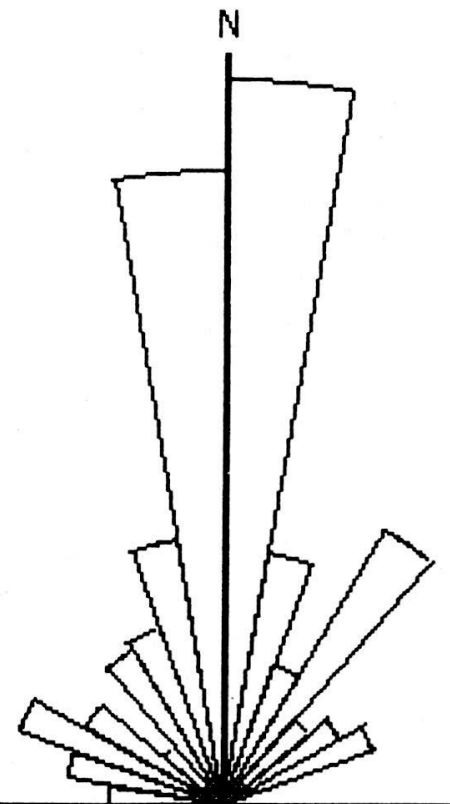

Fig. 8 - Direzioni dei segmenti dello schizzo precedente scomposti in unità elementari ciascuna della lunghezza equivalente a circa $50 \mathrm{~cm}$ sul terreno, ( 773 unità, equivalenti complessivamente a $386,5 \mathrm{~m}$, di cui 315 unità appartenenti a vaschette, le rimanenti a solchi). Nel diagramma stellare del tipo ad aree proporzionali (la scala lungo i raggi equivale alla radice quadrata della percentuale) si può notare come la prevalenza delle direzioni sia compresa fra $170^{\circ}$ e $190^{\circ}$, e come tuttavia siano riconoscibili altre direzioni "oblique" fra cui spicca la $210^{\circ}-220^{\circ}$.

Dai grafici risulta la variabilità delle forme e la debole interrelazione fra diversi parametri quali lunghezza e larghezza, larghezza e profondità. Dalla tabella risulta la prevalenza di forme particolari $(53,26 \%)$ rispetto alle ellittiche $(18,48 \%)$, circolari $(14,67 \%)$ e a goccia $(13,59 \%)$. Per quanto riguarda il fondo invece predominano nettamente $\mathrm{i}$ fondi piatti $(60,44 \%)$, rispetto a quelli che mostrano microversanti e crestine $(20,33 \%)$, ed a quelli irregolari $(19,23 \%)$; fra i fianchi prevalgono quelli subverticali $(57,61 \%)$, rispetto a quelli strapiombanti o aggettanti $(36,41 \%)$, ed a quelli poco inclinati $(5,98 \%)$.

Relativamente agli assi maggiori e ai primi tratti dei canali di scarico prevalgono le direzioni comprese fra $180^{\circ}-240^{\circ}$ (equivalente come orientamento allo $0^{\circ}-60^{\circ}$ ). Nel grafico che mostra le interrelazioni fra la direzione degli assi maggiori e le lunghezze delle kamenitze, si nota che il campo di distribuzione ha una forma a campana (le due metà della campana sono scomposte in modo speculare sui due lati del grafico; a tale proposito si consideri come dal punto di vista dell'orientmento il valore $0^{\circ}$ 
equivale al $180^{\circ}$ ), la quale evidenzia come le vasche più lunghe presentino orientamenti entro il settore fra $\mathrm{N} 20^{\circ} \mathrm{W}\left(340^{\circ}\right)$ e N20 ${ }^{\circ} \mathrm{E}\left(20^{\circ}\right)$ (Fig. 6).

Le kamenitze, per lo più raggruppate in zone a maggior densità (sono in gruppo 1' $89 \%$ delle vaschette contro un $11 \%$ di forme isolate), presentano una lunghezza media intorno a $50 \mathrm{~cm}$, con estremi a $7 \mathrm{~cm}$ ed a $360 \mathrm{~cm}$, ed una profondità media di $7 \mathrm{~cm}$, con estremi di $2 \mathrm{~cm}$ e di $60 \mathrm{~cm}$. Se ne può dedurre che praticamente mancano o sono molto scarse le vere forme embrionali quali le coppellette. La maggior parte delle forme sono invece delle forme composite (69\%) del tipo a vasca talora lobata e policiclica, generalmente aperta $(95,65 \%$ di forme aperte). Solo il $4,35 \%$ delle forme sono del tipo chiuso. Tuttavia in nessun caso si avvicinano al tipo a coppa.

Dagli schizzi geomorfologici a grandissima scala si colgono alcuni interessanti aspetti relativi alle interrelazioni fra le forme (Fig. 7).

In particolare se ci soffermiamo ad analizzare lo schizzo relativo all'affioramento C' si nota come vaschette e solchi formino insieme un reticolo di tipo idrografico in cui la maggior parte dei segmenti sono diretti secondo la massima pendenza delle superfici (intorno a $180^{\circ}$ ), che corrisponde all'immersione degli strati. Se si schematizza questo reticolo trasformando in segmenti sia i solchi, sia gli assi maggiori delle vaschette, sia altre direzioni di sviluppo di queste riconoscibili dalla morfologia (assi di vaschette ormai fuse con altre vasche contigue, direzioni di allungamento in corrispondenza di emissari secondari, ecc.) si ottengono reticoli tanto più gerarchizzati quanto più ampio è l'affioramento. Se si assegna una gerarchia ai diversi segmenti, si giunge ad assegnare sino al terzo ordine idrografico.

Inoltre se si esaminano le direzioni dei segmenti dopo averli scomposti in unità elementari ciascuna della lunghezza equivalente a circa $50 \mathrm{~cm}$ sul terreno, si ottengono circa 760 unità, equivalenti complessivamente a $386 \mathrm{~m}$, di cui 315 unità (circa $157,5 \mathrm{~m}$ ) appartenenti a vaschette e le rimanenti a solchi. In diagramma stellare ad aree proporzionali (la scala lungo i raggi equivale alla radice quadrata della percentuale) si può notare come la prevalenza delle direzioni sia compresa fra $170^{\circ}$ e $190^{\circ}$, e come tuttavia siano riconoscibili altre direzioni "oblique" fra cui spicca la $210^{\circ}-220^{\circ}$.

Da una lettura dello schema si può rilevare come i segmenti obliqui, che molto spesso contraddistinguono le convergenze idrografiche, si trovino allineati lungo fasce all'incirca perpendicolari all'immersione $\left(75^{\circ}\right.$ $255^{\circ}$ ) fra le quali è particolarmente evidente la fascia situata circa 5-6 m a valle del margine superiore del campo solcato. Oltre a questa disposizione per fasce, si può osservare, per alcuni segmenti, una disposizione a formare lineazioni, ben evidente per l'orientamento circa $110^{\circ}-290^{\circ}$. 


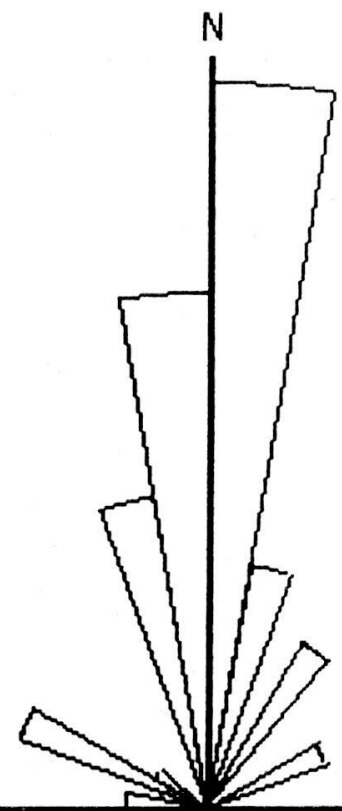

Fig. 9 - Direzioni dei segmenti dello schizzo precedente relativi agli assi delle vaschette scomposti come nel grafico di fig. 8 .

\section{CONSIDERAZIONI CONCLUSIVE ED IPOTESI SULLA STORIA DEI CAMPI SOLCATI}

A questo punto, nell'ambito del problema dell'interpretazione genetica ed evolutiva di questi campi solcati, si possono porre, sia pure in via preliminare, i seguenti quesiti:

- perché, e a partire da quale tipo di rilievo si sono individuate le microcueste in roccia?

- qual è l'età dei campi solcati?

- quali fattori hanno condizionato lo sviluppo e l'impostazione dei diversi tipi di forme su questi campi solcati?

I campi solcati occupano una "superficie di erosione", che tronca in modo netto le strutture geologiche. Si tratta di una superficie di spianamento sui cui problemi genetici non ci vogliamo addentrare, limitandoci a rimandare alla bibliografia sia generale sia specifica su questo aspetto (CASTIGLIONI et alii, 1989,).

Nell'ambito di questa superficie di erosione le bancate più resistenti possono essere messe in evidenza da una degradazione selettiva di tipo sia 
fisico, sia chimico. In realtà la sola corrosione carsica non spiegherebbe una degradazione differenziale in quanto tutti i calcari puri sono soggetti alla corrosione indipendentemente dalla loro resistenza e nemmeno sembra sufficiente invocare la "corrosione diffusa" nelle fasce di roccia più minutamente fratturata. È bene notare come questi affioramenti rocciosi mostrino una più elevata concentrazione in prossimità dei bordi delle doline $\mathrm{e}$ come quindi le depressioni monoclinali comprese fra i rilievi in roccia possano essere anche ritenute l'espressione di una corrosione accelerata in relazione ad un deflusso verso la conca determinato da un aumento della conduttività idraulica. In altre parole le depressioni monoclinali fra i rilievi potrebbero rappresentare "solchi" " da corrosione accelerata.

Non si può però tralasciare il ruolo dei processi della degradazione fisica ed in particolare di quelli clastici la cui efficacia si è espressa nell'individuazione di alcune "tavole" o "funghi". Questi processi sono stati senza dubbio particolarmente attivi durante le ultime fasi fredde del Pleistocene, quando qui si estendeva una steppa fredda nella quale si depositavano coltri di limi loessici. I processi crioclastici favorivano la disgregazione delle bancate più gelive e quindi la sottoescavazione di alcune paretine in roccia (scarpate di testata delle microcueste e paretine delle tavole).

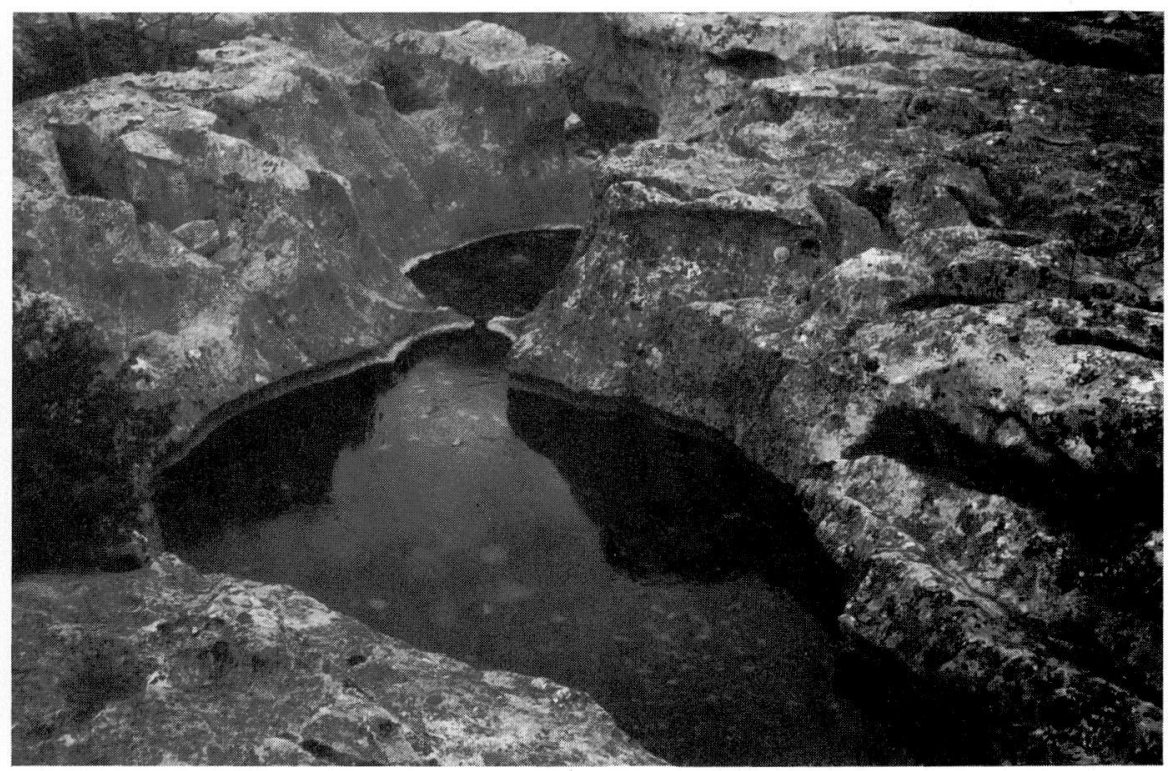

Fig. 10 - Kamenitze giganti, affioramento C. 


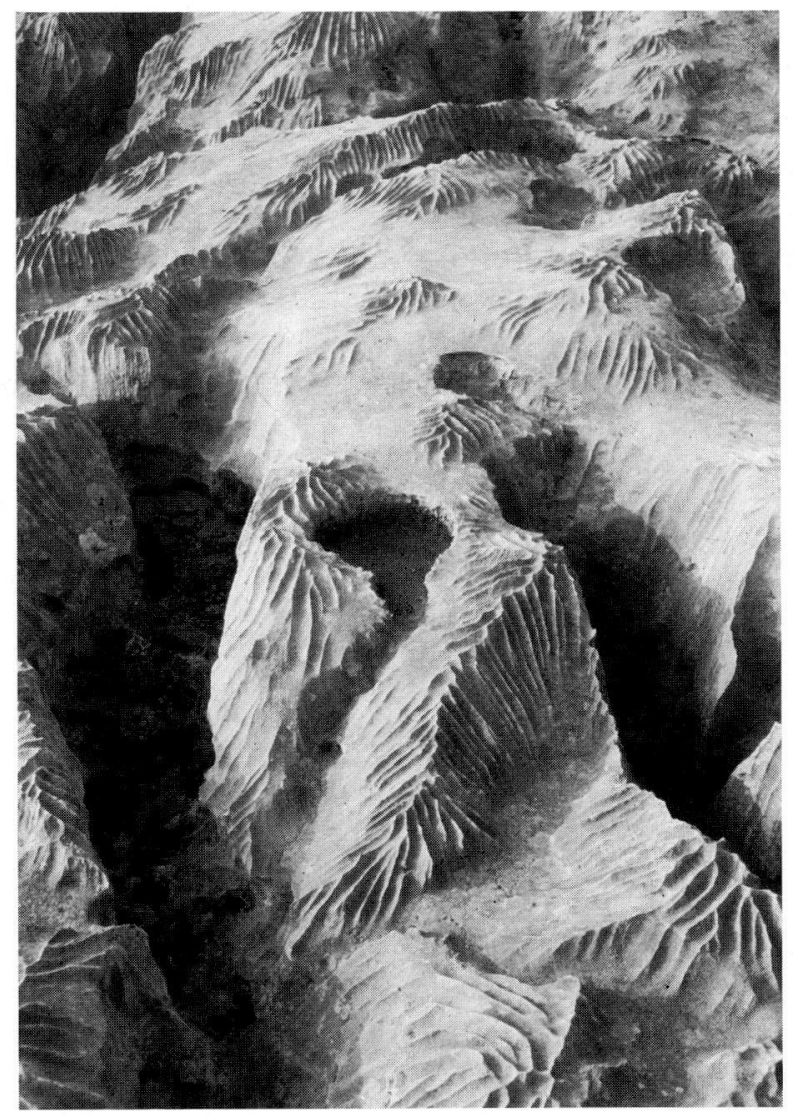

Fig. 11

Campo solcato, affioramento C.

Relativamente ai campi solcati viene da chiedersi se questi rappresentino l'espressione di un'evoluzione in atto già dal Pleistocene superiore ed in particolare dall'ultima fase fredda (Würm) o se siano complessi morfologici più recenti. Quest'ultima sembra l'ipotesi più plausibile in quanto $i$ campi solcati su superfici di rocce montonate delle valli alpine meridionali mostrano all'incirca lo stesso grado di sviluppo, anche se stili ed aspetti diversi (SAURO, 1973; PERNA \& SAURO 1978).

Se si considerano le forme prevalenti e le loro associazioni si può ipotizzare che alla fine del Pleistocene e all'inizio dell'Olocene le microcueste fossero in gran parte ricoperte da limi loessici su cui si sviluppava un suolo di tipo forestale. Al contatto roccia-coperture si andava individuando un "campo solcato" di tipo coperto con Karren arrotondati. In seguito al disboscamento provocato dall'uomo e all'erosione del suolo determinata 
dalla pastorizia e facilitata dalla vicinanza della dolina, molte superfici sono passate da condizioni di tipo coperto a condizioni di tipo libero. Sui Karren di tipo arrotondato si sono sovraimposti Karren di tipo libero o semilibero quali i solchi a doccia e le vaschette carsiche. Potrebbe essere proprio il microrilievo dei Karren di tipo arrotondato a spiegare alcuni degli aspetti evolutivi del campo solcato. In particolare le fasce con segmenti di direzione obliqua potrebbero corrispondere ad antichi "limiti delle coperture di suolo", ove la corrosione era relativamente accelerata con individuazione di depressioni trasversali che hanno creato ondulazioni modificando localmente le condizioni di pendenza.

Che la situazione della copertura di suolo non sia sempre stata quella attuale lo dimostrano, oltre ai fenomeni di erosione attuale in aree limitrofe ove vengono man mano alla luce forme "coperte", la presenza di più cornici all'interno di alcune vaschette. Si tratta di cornici che sembrano documentare un'alternanza di fasi con un'evoluzione di tipo libero (vaschette con sola acqua all'interno) e di fasi con un'evoluzione di tipo semilibero (vaschette con zolle di suolo all'interno che provocano anche un abbassamento della soglia in roccia) (SAURO, 1973, 1974).

Considerando infine la percentuale occupata da forme di tipo diverso nei due campi solcati esaminati sembra che il grado di evoluzione raggiunto sia all'incirca il medesimo (occupazione delle superfici per oltre un terzo e per circa un quinto da vaschette e solchi insieme). Con il tempo il microrilievo dovrebbe aumentare a causa dell'approfondimento di alcune forme e della comparsa di fori e crepacci; nel contempo aumenta la percentuale occupata da scannellature, sino ad una scomposizione in blocchi con formazione di grize, cioè di campi di detriti rocciosi. Altrove, ove le condizioni litologiche e strutturali erano diverse, molti campi solcati hanno già raggiunto lo "stadio" di griza. Sulle grize è più facile l'impianto di un suolo vegetale.

Senza dubbio questi caratteristici paesaggi del Carso offrono ancora spunti per ulteriori studi di tipo interdisciplinare e, rappresentando dei singolari monumenti naturali, sono meritevoli di protezione. I loro caratteri di micropaesaggi in evoluzione, risultanti da particolari interazioni alle interfacce roccia/suolo/atmosfera, li rende non solo interessanti dal punto di vista scientifico, ma anche stimolanti nell'ottica di un auspicato sviluppo di una didattica naturalistico-ambientale. Ben vengano quindi in quest'area iniziative rivolte alla creazione di riserve di monumenti naturali con sentieri attrezzati per un turismo di tipo culturale. 
TABELLE RELATIVE A PARAMETRI MORFOMETRICI DI 184 VASCHETTE DI CORROSIONE DEI CAMPI SOLCATI DI BORGO GROTTA GIGANTE NEL CARSO TRIESTINO.

NELLA PRIMA PAGINA SONO ELENCATI I PARAMETRI MORFOMETRICI E AMBIENTALI CONSIDERATI.

\section{VASCHETTE CARSICHE}

No : numeri progressivi delle vaschette

Aff. $\quad$ : sigle degli affioramenti $\left(\mathbf{A}, \mathbf{B}, \mathbf{C}\right.$ e $\left.\mathbf{C}^{\prime}\right) \quad(.:$ vaschette composite $)$

\begin{tabular}{|c|c|c|c|}
\hline Rel. & : & 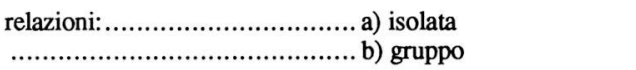 & $\begin{array}{l}\text { (is.) } \\
\text { (gr.) }\end{array}$ \\
\hline Ass. & : & 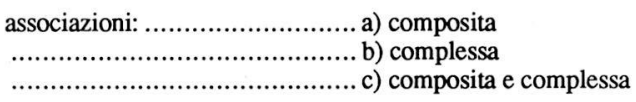 & $\begin{array}{l}(\mathrm{cs} .) \\
(\mathrm{cl} .) \\
(\mathrm{cs} . \mathrm{e} \mathrm{cl} .)\end{array}$ \\
\hline Tipi & : & tipi: & $\begin{array}{l}\text { (ch.) } \\
\text { (ap.) }\end{array}$ \\
\hline For. & : & 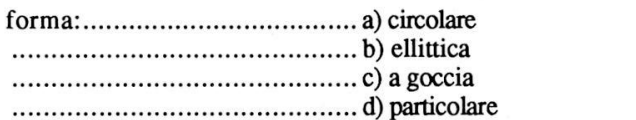 & $\begin{array}{l}\text { (cir.) } \\
\text { (ell.) } \\
\text { (a g.) } \\
\text { (par.) }\end{array}$ \\
\hline
\end{tabular}

Lun. $\quad$ : lunghezza (L.)

Dir. $\quad$ : direzione di L. $\left(0^{\circ}-180^{\circ}\right)$

Lar. : larghezza (1.)

Prof. : profondità (p.)

P.m. : profondità massima

L.b : lunghezza di (b)

1.b. : larghezza di (b)

p.b : profondità di (b)

S.1 : Cornice 1

S.2 : Comice 2

S.3 : Comice 3

Fon. $\quad$ : fondo:................................ a) piatto

(irr.)
(m...)

Car.f. : caratteristiche del fondo: ............ a) detriti rocciosi (d.)

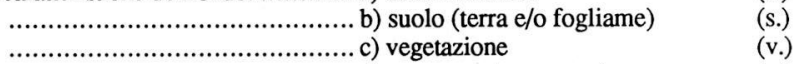
................................... d) acqua semipermanente (a.) e) nudo

Par. $\quad$ : pareti:............................. a) poco inclinate $\left(<60^{\circ}\right) \quad$ (p.i.) .................................... b) subverticali (sbv.)

c) aggettanti (agg.)

D.c. : direzione del canale di scarico $\left(0^{\circ}-360^{\circ}\right)$ 




âj.

को

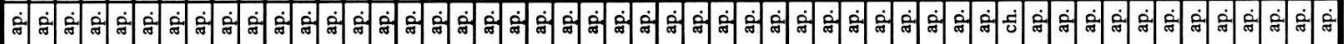

安

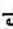

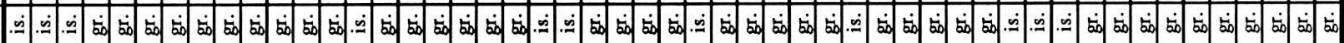

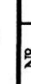

\& 


\begin{tabular}{ll}
\hline \\
\hline
\end{tabular}

:

宊

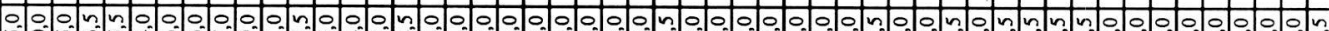
(1)

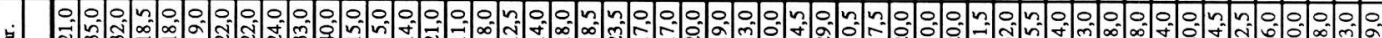
का लेख 熍

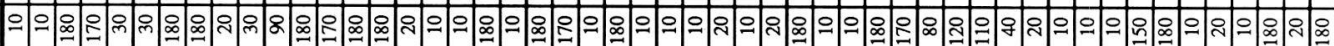

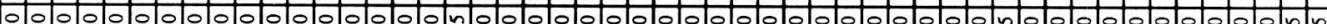
1)


$\vec{g}$

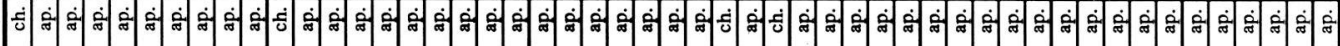
安 $\dot{\vec{\Xi}}$

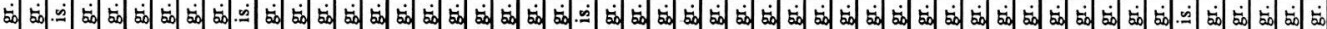

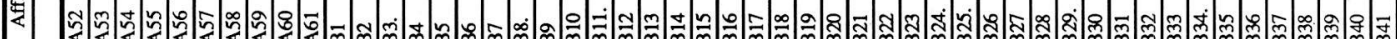

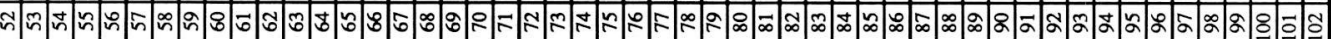

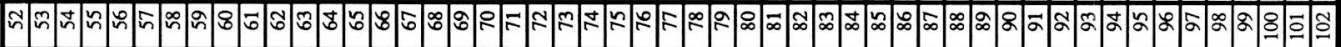




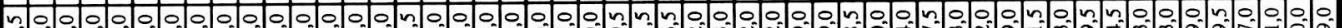

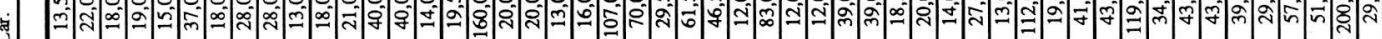

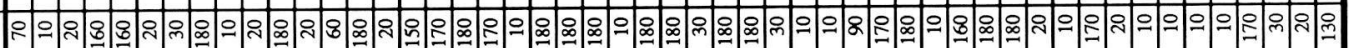

西

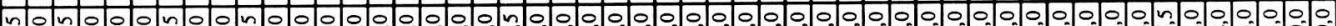

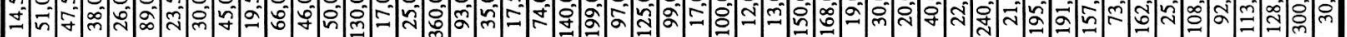

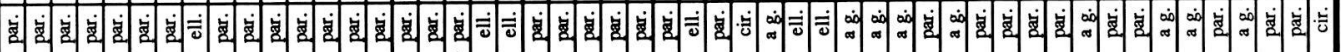

के

?

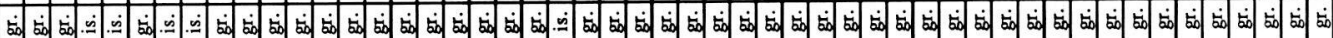

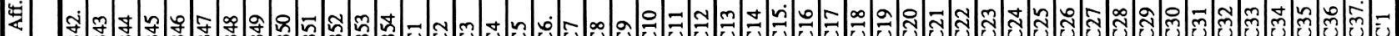

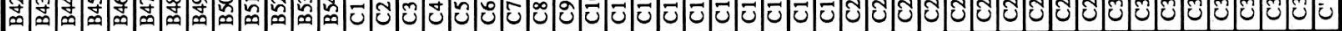

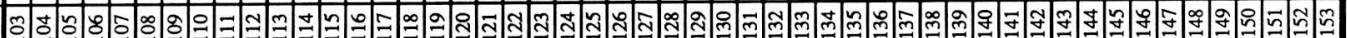

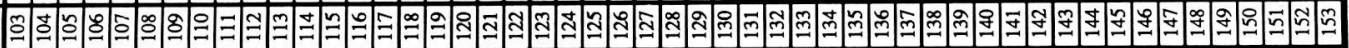




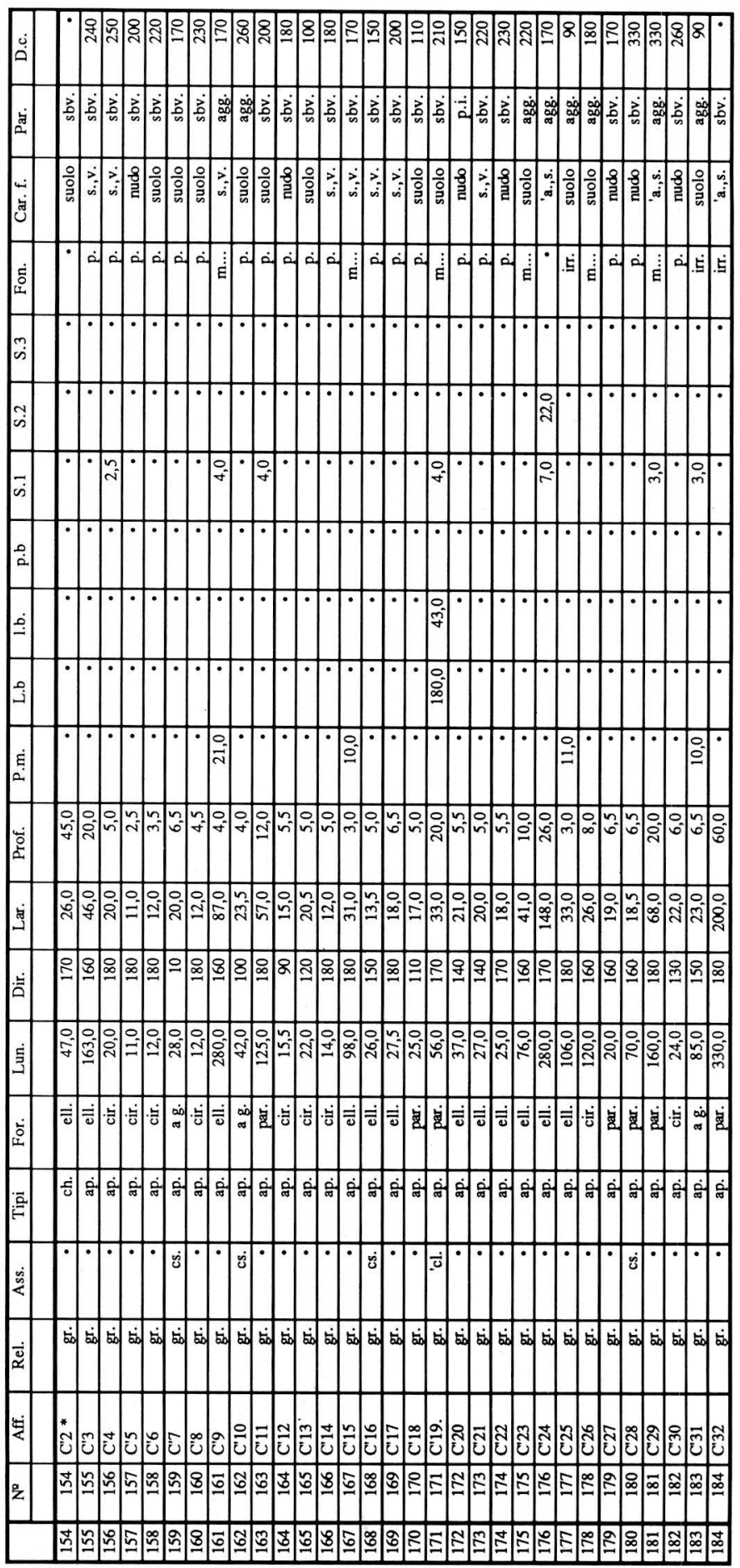




\section{BIBLIOGRAFIA}

Belloni S. \& Orombelli G. 1970: Osservazioni e misure su alcuni tipi morfologici nei campi solcati del Carso Triestino. Atti Soc. It. Sc. Nat. e Museo Civ. St. Nat. Milano, v. 110, 317-372.

BELLONI S. 1969: Alcune osservazioni sulle acque e sui depositi al fondo delle "vaschette di corrosione" (Kamenitza) della località Borgo Grotta Gigante del Carso Triestino. Atti e Memorie Comm. Grotte "E. Boegan”, v. 9, 33-62.

Bini A., Meneghel M. \& SAuro U. 1986: Proposta di legenda per una cartografia geomorfologica delle aree carsiche. Atti e Memorie Comm. Grotte 'E. Boegan', v. 25, 2129.

BOEGLI L.A. 1960: Kalklösung and Karrenbildung. Zeitschr. für Geomorphologie, s. 2, 4-21.

CANCIAN C. 1984: I "solchi di dissoluzione" (Rinnenkarren) negli alti e bassi Carsi del Friuli-Venezia Giulia. Studi Trentini Sc. Nat., A.G., 61, 101-112.

CAstiglioni G.B., Meneghel M. \& SAURo U. 1989: Elementi per una ricostruzione dell'evoluzione morfotettonica delle Prealpi Venete. Riv. Geografica Fis. Din. Quatern. suppl. 1 (1988), 31-44.

CUCCHI F., FORTI F. \& UlCIGRAI F. 1976: Relazioni fra tettonica e morfogensesi di doline del Carso Triestino e Monfalconese. Atti e Memorie Comm. Grotte “E. Boegan", v. 15, 1976, 57-71.

CUCCHI F., FORTI F. \& FORTI P. 1983: Movimenti recenti nel Carso Triestino da analisi di concrezioni stalagmitiche. Geogr. Fis. Dinam. Quat., 6 (1983), 43-47.

Cucchi F. \& Pugliese N. 1987: Il Carso Triestino: geologia e stratigrafia. Giornate di Seminari: Maria Luisa Zucchi Stolfa. Università di Trieste (in stampa).

Cucchi F., Pirini Radrizzani \& Pugliese N. 1987: The Karst of Trieste. Guidebook of excursion 4th June 1987. Int. Symp.: "Evolution of the karstic Carbonate Platform: relation with other periadriatic carbonate platforms. Trieste, $32 \mathrm{pp}$.

DE FANTI A. 1971: Forme di corrosione dovute a microorganismi osservate nel Gruppo del Civetta (Alpi Dolomitiche). Boll. Soc. Geogr. It. 9/12, 605-612.

FolK L.R., RoberTs H.H. \& MoORE C.H. 1973: Black phytokarst from Hell Cayman Islands, British West Indies. Bull. Geol. Soc. of America, 84/7, 2351-2360.

FORD. D.C. \& LUNDBERG J.A. 1987: A review of dissolutional rills in limestone and other soluble rock. Catena S. 8, 119-140.

FORD D.C. \& WiLliams P. 1989: Karst geomorphology and hydrology. Unwin Hyman, $601 \mathrm{pp}$.

FORTI F. 1971: Le "vaschette di corrosione". Rapporti fra geomorfologia carsica e condizioni geolitologiche delle carbonatiti affioranti sul Carso Triestino. Atti e Memorie Comm. Grotte "E. Boegan”, v. 11, 37-65.

FORTI F. 1972: Studio geomorfologico dei "Fori di dissoluzione" nelle carbonatiti calcaree del Carso Triestino. Atti e Memorie Comm. Grotte "E. Boegan", v. 12, 19-28.

FORTI F. 1979: Dissoluzione sottocutanea accelerata nelle doline del Carso Triestino. Atti e Memorie Comm. Grotte “E. Boegan”, v. 19, 73-78. 
Gavrilovic D. 1968: Kamenice - Kleine Korrosionsformen im Kalkstein. Proc. $4^{\circ}$ Int. Speleolog. Congress., Ljubliana, Yugoslavia, v. 3, 127-133.

PERNA G. 1974: Il fitocarsismo nella formazione delle Kamenitze (Vaschette di corrosione). Natura Alpina, v. 15, 25-34.

PERna G. \& SAURo U. 1978: Atlante delle microforme di dissoluzione carsica superficiale del Trentino e del Veneto. Mem. Museo Tridentino di Scienze Nat., 22, 176 pp.

Pluhar A. \& Ford D.C. 1970: Dolomite Karren of the Niagara Escarpment, Ontario Canada. Zeitschr. für Geomorphologie, 14/4, 392-410.

Radovich N. 1988: I Karren di Borgo Grotta Gigante (Carso di Trieste). Tesi di Laurea inedita in Scienze Naturali. Relatori U. Sauro e F. Cucchi Univ. di Padova, Dip. di Geografia, 86 pp.

SAURO U. 1973: Observations on some great solution runnels with nested pans of the Venetian Prealps. Proc. 6th Int. Speleol. Congr., Olomouc, Ba 039, 353-361.

SAURO U. 1974: Forme di corrosione su rocce montonate nella Val Lagarina meridionale. L'Universo, 53, 2, 309-344.

SAURO U. 1975: The geomorphological mapping of "Karrenfelder" using very large scales: an example. Karst Processes and relevant landforms, I. Gams Ed., 189-199, Ljubljana.

SAURO U. 1977: Proposte per una cartografia geomorfologica a grandissima scala dei campi solcati (lapiés). Studi Trentini di Sc. Nat., A.G., 54, 163-176.

Sauro U. 1979: Morfologia carsica. In “Geomorfologia”, G.B. Castiglioni Ed., UTET, 208-254

SAURO U. 1982: Materiali e problemi per uno studio paleogeografico delle regioni carsiche italiane. Lav. Soc. Italiana di Biogeografia, n. s. 7, 1978, 467-513.

VILES H.A. 1984: Biokarst: review and prospéct. Progress in Physical Geography, v. 8, f. 4, 523-542. 